\title{
The Formation of Constellation III in the Large Magellanic Cloud
}

\author{
Jason Harris $^{\mathrm{A}, \mathrm{B}}$ and Dennis Zaritsky ${ }^{\mathrm{A}}$ \\ A Steward Observatory, 933 N. Cherry Ave., Tucson, AZ 85721, USA \\ ${ }^{\text {B } C o r r e s p o n d i n g ~ a u t h o r . ~ E m a i l: ~ j h a r r i s @ ~ a s . a r i z o n a . e d u ~}$
}

Received 2007 September 20, accepted 2007 December 12

\begin{abstract}
We present a detailed reconstruction of the star-formation history of the Constellation III region in the Large Magellanic Cloud, to constrain the formation mechanism of this enigmatic feature. Star formation in Constellation III seems to have taken place during two distinct epochs: there is the 8-15 Myr epoch that had previously been recognized, but we also see strong evidence for a separate 'burst' of star formation 2530 Myr ago. The 'super-supernova' or GRB blast wave model for the formation of Constellation III is difficult to reconcile with such an extended, two-epoch star formation history, because the shock wave should have induced star formation throughout the structure simultaneously, and any unconsumed gas would quickly be dissipated, leaving nothing from which to form a subsequent burst of activity. We propose a 'truly stochastic' self-propagating star formation model, distinct from the canonical model in which star formation proceeds in a radially directed wave from the center of Constellation III to its perimeter. As others have noted, and we now confirm, the bulk age gradients demanded by such a model are simply not present in Constellation III. In our scenario, the prestellar gas is somehow pushed into these large-scale arc structures, without simultaneously triggering immediate and violent star formation throughout the structure. Rather, star formation proceeds in the arc according to the local physical conditions of the gas. Self-propagating star formation is certainly possible, but in a truly stochastic manner, without a directed, large scale pattern.
\end{abstract}

Keywords: galaxies: magellanic clouds — galaxies: stellar content

\section{Introduction}

Shapley's Constellation III in the Large Magellanic Cloud (LMC) is one of the most enigmatic structures in the local universe: a coherent semicircular arc spanning several hundred parsecs, composed of thousands of bright young stars and tens of star clusters. Its regularity across such a large scale defies the fractal-like distributions that young stellar populations typically follow; in fact, Constellation III may be unique in this regard. In addition, Constellation III is embedded inside the supergiant shell LMC 4, a circular hole in the LMC's Hi disk that spans more than a kiloparsec (Kim et al. 1999), and whose rim is dotted with HII regions (Meaburn 1980).

The singular nature of Constellation III invites speculation about its formation mechanism, which must have been similarly unique given the absence of anything resembling this structure in other nearby galaxies. Westerlund \& Mathewson (1966) popularized the 'Constellation III' designation, and speculated that its stars were formed from material swept up in the shock of a 'super-supernova'. Efremov \& Elmegreen (1998) suggested that the combined winds from a relatively small number of massive stars could have swept LMC4 clean of gas, and subsequently triggered the formation of Constellation III. However, as they point out in Efremov \& Elmegreen (1999), the unique nature of Constellation III belies such a mundane formation mechanism. After all, the LMC is home to thousands of star clusters which must have hosted similarly strong massive-star winds, yet it contains no other structures like Constellation III. Instead, Efremov \& Elmegreen (1999) favor an updated 'supersupernova' idea, suggesting the LMC4 cavity was blown by a gamma-ray burst formed by the coalescence of an $\mathrm{X}$-ray binary (perhaps ejected from the nearby rich star cluster NGC 1978), and the swept-up material subsequently formed Constellation III. Dopita, Mathewson \& Ford (1985) presented the idea that Constellation III is the result of a stochastic self-propagating star formation (SSPSF) process, directed in an outward radial propagation. However, recent studies of the distribution of ages in Constellation III (Olsen et al. 1997; Braun et al. 1997; Dolphin \& Hunter 1998) have not confirmed the radial age gradient reported by Dopita et al. (1985).

In this paper, we present a map of the past history of star formation in the vicinity of Constellation III, in order to constrain the various formation scenarios for this unique structure. In Section 2, we briefly review the photometric data and our STARFISH analysis software. In Section 3, we present a detailed map of the star formation history (SFH) throughout the Constellation III region. We discuss the implications of our SFH map in Section 4, and summarize the results in Section 5. 


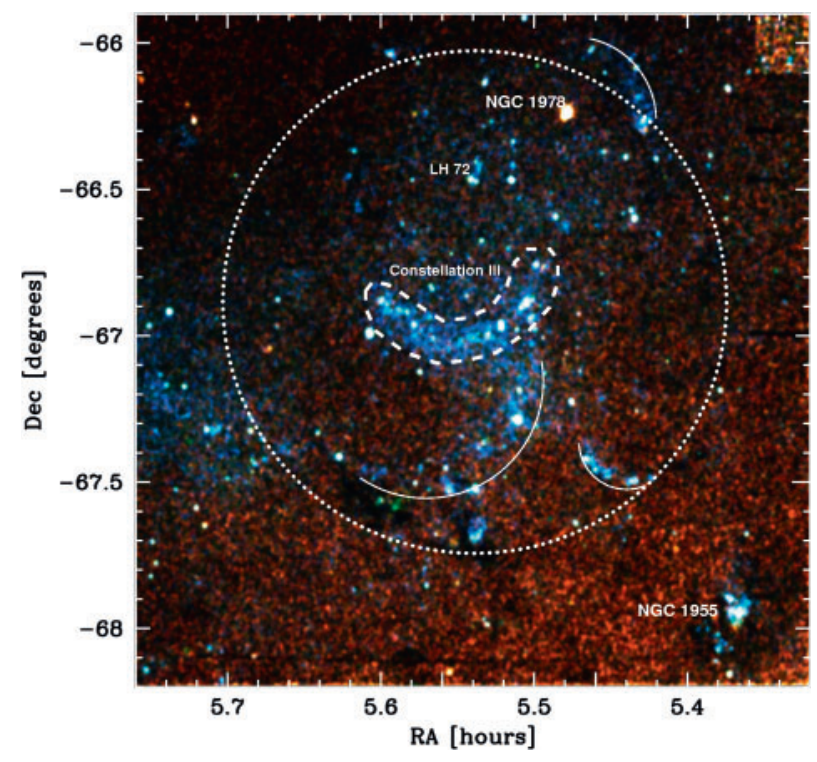

Figure 1 A stellar flux density map of a $2.5 \times 2.5$-deg region in the LMC, including Constellation III. The map was derived from our MCPS photometry: each pixel's value is proportional to the total stellar flux in $B, V$ and $I$ (for blue, green and red, respectively). Major structures and clusters are labeled, including Constellation III itself (dashed outline), and the approximate position of the LMC 4 supergiant shell (large dotted circle). Note that Constellation III is actually one of a few large stellar arcs in this region; our analysis will include all of these arcs.

\section{Overview of the Magellanic Clouds Photometric Survey and STARFISH}

The Magellanic Clouds Photometric Survey (MCPS, Zaritsky et al. 2002, 2004) is a drift-scan survey of both the LMC and the Small Magellanic Cloud (SMC), undertaken at the Las Campanans Observatory 1-meter Swope telescope between 1995 and 2000. The MCPS provided CCD imaging to $V=21 \mathrm{mag}$ in $U, B, V$, and $I$ filters, covering $8.5 \times 7.5 \mathrm{deg}$ in the LMC, and $4 \times 4.6 \mathrm{deg}$ in the SMC. Our catalogs contain astrometry and photometry for 24 million LMC stars and more than 6 million SMC stars.

In Figure 1, we show a stellar flux density map derived from our MCPS photometry, for a $2.5 \times 2.5$-deg region including Constellation III. The Figure shows that the arc traditionally known as Constellation III is actually one of at least three large stellar arcs in this region; all of these arcs lie in the interior of the LMC $4 \mathrm{HI}$ supergiant shell.

The stellar populations of a galaxy represent a fossil record of its past star-formation activity. By statistically comparing multicolor photometry of resolved stellar populations to synthetic populations based on theoretical isochrones (e.g. Girardi et al. 2002), we can reconstruct the SFH of the target galaxy. We have developed a software package (STARFISH, Harris \& Zaritsky 2001), which performs robust SFH analysis of resolved stellar photometry. STARFISH works by constructing a library of synthetic color-magnitude diagrams (CMDs), each of which is built from isochrones spanning a small range in age and metallicity. The synthetic CMDs are designed to replicate the

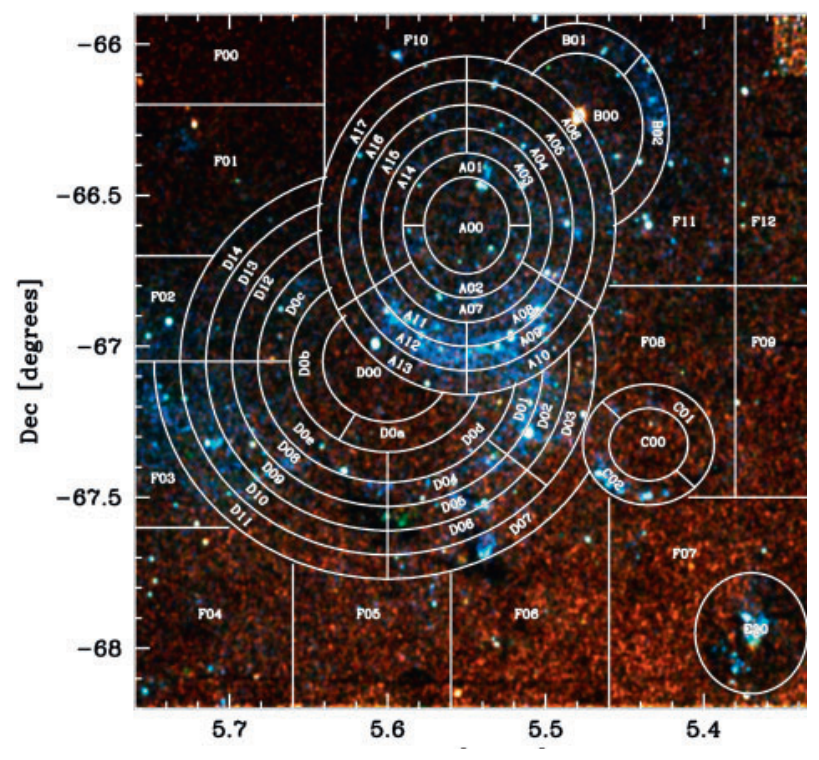

Figure 2 The same flux-density maps as in Figure 1, with our conformal grid overplotted. We determined the best-fit SFH solution independently for each grid cell in order to generate a map of the $\mathrm{SFH}$ in this region.

characteristics of the observed data in every way (distance, extinction, IMF, binarity, photometric errors, and incompleteness). Each synthetic CMD therefore represents the contribution to the CMD of stars of a particular age and metallicity. Through linear combination of synthetic CMDs spanning all relevant combinations of age and metallicity, we can generate composite model CMDs that represent any arbitrary SFH. These composite model CMDs can then be quantitatively compared to the real, observed CMD, and by minimizing the differences between them, the best-fitting SFH solution can be obtained.

\section{Mapping the SFH of the Constellation III Region}

In order to distinguish between the various competing theories for the formation of Constellation III, we constructed a spatially resolved map of the SFH of the entire $2.5 \times$ 2.5-deg region. In order to maximize our ability to detect any radial or azimuthal population gradients present in these stellar arcs, we constructed a conformal grid that follows their curvature (see Figure 2), and determined an independent SFH solution for the stars in each grid cell. The synthetic CMDs employed for each grid cell used extinction distributions and empirical photometric error models derived directly from the grid cell's stellar population.

Previous analyses of the SFH of Constellation III have largely sought to determine a single characteristic age for different locations in the structure, without considering the full distribution of stellar ages that makes up the true SFH. In particular, the ages assigned have been the youngest age present. As an illustration that a more complete $\mathrm{SFH}$ is warranted for these regions, we examine the $(B-V)$ 


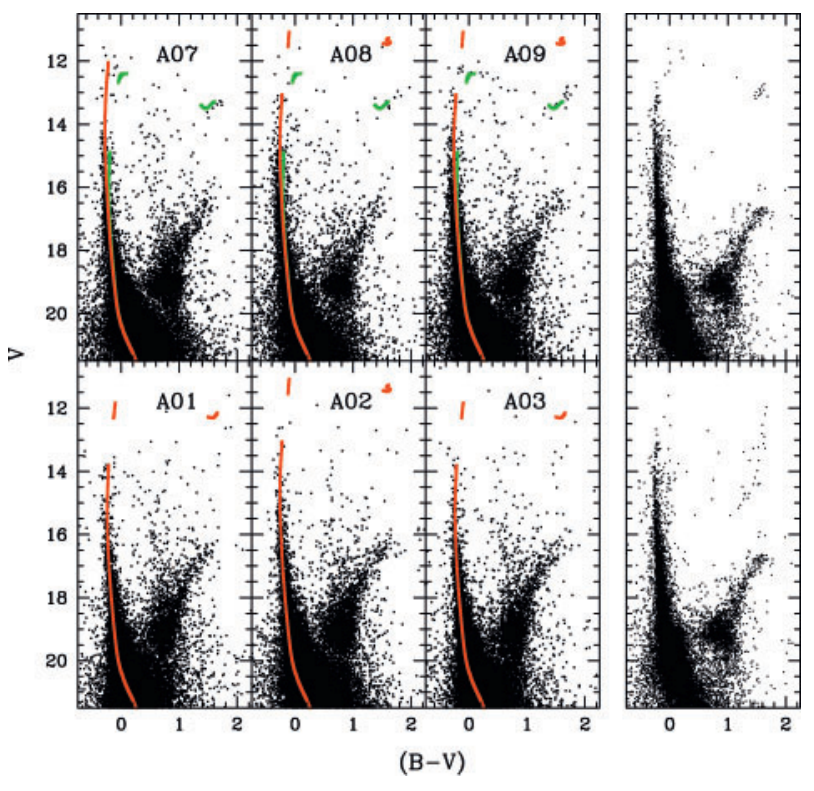

Figure 3 The six panels on the left show $(B-V)$ vs. $V$ CMDs for six of our Constellation III regions (as labeled at the top of each panel; see Figure 2). The termination of the main sequence at $V=12-13$ mag indicates that the youngest stars in these regions is aged 10-15 Myr (the red isochrones overplotted in each panel). In addition, the three populations in the top row show an isolated clump of red supergiants near $V=13.5 \mathrm{mag}$, indicative of a burst of star formation activity around $30 \mathrm{Myr}$ ago (the green isochrones overplotted in the top panels). This is further illustrated by the synthetic stellar populations in the rightmost column. These two populations differ only in their SFH between 10 and $100 \mathrm{Myr}$ : in the top panel, the stars in this age range all have an age of $30 \mathrm{Myr}$, whereas in the bottom panel, the ages are uniformly distributed between 10 and $100 \mathrm{Myr}$.

CMDs of eight of our Constellation III regions in Figure 3. Each of these regions shows a prominent main sequence, and by simple isochrone fitting, one can determine the age of the youngest stellar population in each region (as shown by the red curves in each panel). However, these CMDs show clear evidence for a more complex SFH. The red giant branch and red clump are obvious tracers of old stellar populations, but there are also supergiants present which trace star formation that occurred several tens of millions of years ago. In particular, the CMDs in the top row of Figure 3 each show an isolated knot of red supergiants with $V=13.5 \mathrm{mag}$. An isolated knot of supergiants at a common luminosity is strong evidence for an isolated burst of star formation activity in the recent history of the region, because the luminosity of a supergiant is directly and unambiguously correlated with its age (Dohm-Palmer et al. 1997). The isochrone overplotted in green in Figure 3 indicates that the supergiants in these knots are roughly $30 \mathrm{Myr}$ old. Thus, by simple visual inspection of these CMDs, we can already conclude that some of the Constellation III regions have experienced multiple, isolated bursts of star formation. Variations like these will be recovered in our STARFISH analysis, giving us a much more complete picture of the SFH of Constellation III.

The SFH map of Constellation III resulting from our STARFISH analysis is shown in Figure 4. In this figure, each panel represents a map of the past star formation rate for a different time step, from $12 \mathrm{Gyr}$ ago to $5 \mathrm{Myr}$ ago. It is immediately apparent from the SFH map that star formation occurred in Constellation III over an extended time interval, from about $30 \mathrm{Myr}$ ago until $8 \mathrm{Myr}$ ago. Star formation was active in different parts of Constellation III at different times; however, there do not appear to be systematic, large-scale age gradients revealed in these maps. We also note that Constellation III does not distinguish itself from the background stellar population until the onset of recent star-formation $30 \mathrm{Myr}$ ago, so it is likely that $30 \mathrm{Myr}$ ago marks the time of its initial formation. This can also be seen in Figure 5, the summed total SFH for the entire Constellation II region

When examining Figure 5, it is important to understand that SFH maps like this do not allow us to display information on the uncertainties associated with the best-fit star formation rates (SFRs) in each time step. However, STARFISH does estimate these uncertainties, and they do include covariance between adjacent age bins. So, for example, in the map there are many regions that have a large SFR in the $10 \mathrm{Myr}$ panel, but a very low SFR in the $8 \mathrm{Myr}$ panel, and vice versa. The uncertainties computed by STARFISH for these bins indicates that these variations are not significant; in other words, it is clear from the fit that there was a large amount of star formation activity $8-10 \mathrm{Myr}$ ago in many of these regions, but in most cases, the data do not allow us to distinguish $8 \mathrm{Myr}$ old stars from $10 \mathrm{Myr}$ old stars, and this non-uniqueness is reflected in the computed uncertainties.

\section{Discussion and Implications}

Most previous analyses of Constellation III's SFH have concluded that the stellar arc is $10-15 \mathrm{Myr}$ old. We have shown that the youngest stars in these arc structures are 10-15 Myr old, but that they also contain abundant populations as old as $30 \mathrm{Myr}$. This extended epoch of star formation is difficult to reconcile with currently proposed ideas about the formation mechanism of Constellation III. In the 'super-supernova' or GRB shockwave scenario, star formation would be triggered throughout the arc structure on a short timescale, resulting in a small age spread among the stars in Constellation III. The SSPSF scenario predicts a more protracted epoch of star formation, but as it is usually discussed in the literature (Dopita et al. 1985; Olsen et al. 1997), the propagation wave is directed radially outward from the center of Constellation III. Our SFH map confirms that there are no large-scale age gradients in Constellation III that would be required by this model.

We propose a new scenario in which the pre-stellar material was swept into large arcs (perhaps by dramatic forces such as a GRB-like explosion, or the combined winds of massive stars), but that star formation was not immediately triggered throughout the structure by these forces. Rather, star formation proceeded stochastically throughout the giant prestellar cloud complex, according to the local physical conditions of the interstellar medium. Self-propagation may well be a part of this process. We 


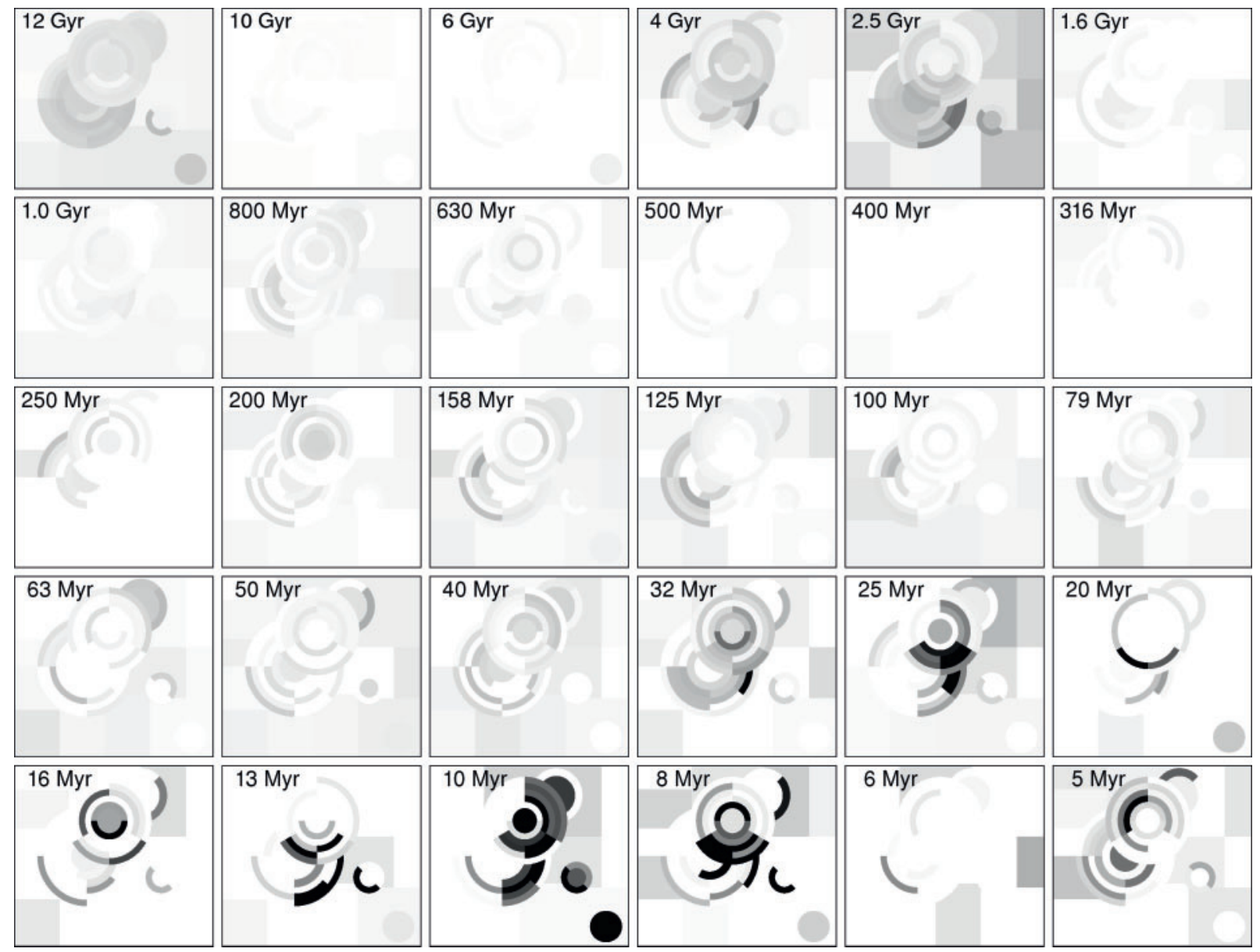

Figure 4 The SFH map for the Constellation III region. Each panel displays the star formation rates for a single time step in the LMC's history, from $12 \mathrm{Gyr}$ ago in the upper left to $5 \mathrm{Myr}$ ago in the lower right. Within a panel, the greyscale is proportional to the relative star formation rate in each grid cell (with darker color corresponding to a larger star formation rate).

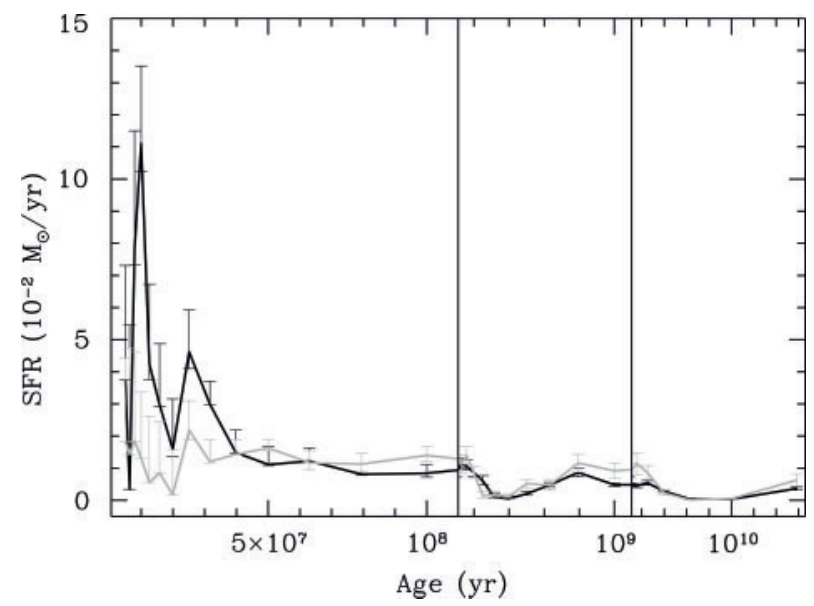

Figure 5 The summed total SFH of the entire Constellation III region, shown as the star formation rate as a function of time. The time axis is displayed linearly, but the scale changes in the three panels so that the very narrow time intervals at the young end can be displayed. The left panel covers about $100 \mathrm{Myr}$, the middle panel covers about $1 \mathrm{Gyr}$, and the right panel covers more than $10 \mathrm{Gyr}$. The black histogram represents the SFH of the stellar arcs in the Constellation III region, while the grey histogram represents the SFH of the background population in this region. do not reject SSPSF per se in the formation of Constellation III, but the large-scale radially directed form in which it is usually discussed for this region.

It may seem unlikely that it would be possible to sweep material into large, coherent structures without triggering massive, rapid star formation in the material. However, the LMC currently contains an example of just such a large, coherent prestellar cloud complex: there is a ridge of molecular gas extending more than $1.5 \mathrm{kpc}$ southward from 30 Doradus, with a typical width of $100 \mathrm{pc}$ (Mizuno et al. 2001). This ridge contains abundant molecular and atomic gas, and yet its specific star formation rate is currently quite low. While the physical processes that led to the formation of this giant molecular ridge may be quite different from the forces that scuplted Constellation III, its existence is evidence that it is at least possible to gather prestellar material into a large coherent structure, without simultaneously triggering rapid star formation throughout it.

\section{Summary}

We present a reconstruction of the spatially resolved SFH of the enigmatic Constellation III region in the northern 
LMC disk. We find that stars in the giant stellar arcs in this region formed over an extended period, from 30 to $10 \mathrm{Myr}$ ago. While there are significant spatial variations in the SFH, there don't appear to be large-scale age gradients as would be expected in a SSPSF formation scenario.

Since our detailed SFH reconstruction of Constellation III fits neither of the widely discussed formation scenarios for this unique structure, we propose a new scenario in which the prestellar material was swept up into large arcs, but star formation was not immediately triggered throughout the cloud, or at least not violently so. The molecular ridge south of 30 Doradus provides evidence that it is possible to organize kpc-scale coherent structures in prestellar material without immediately triggering rapid star formation in the gas.

\section{Acknowledgments}

Much of this work was performed while JH was supported by NASA through Hubble Fellowship grant HF-01160.01A awarded by the Space Telescope Science Institute, which is operated by the Association of Universities for Research in Astronomy, Inc., under NASA contract NAS 5-26555. DZ acknowledges financial support from National Science Foundation grant AST-0307482 and a fellowship from the David and Lucille Packard Foundation.

\section{References}

Braun, J. M., Bomans, D. J., Will, J.-M. \& de Boer, K. S., 1997, A\&A, 328, 167

Dohm-Palmer, R. et al., 1997, AJ, 114, 2527

Dolphin, A. E. \& Hunter, D. A., 1998, AJ, 116, 1275

Dopita, M. A., Mathewson, D. S. \& Ford, V. L., 1985, ApJ, 297, 599

Efremov, Y. N. \& Elmegreen, B. G., 1998, MNRAS, 299, 643

Efremov, Y. N. \& Elmegreen, B. G., 1999, in IAU Symposium Vol. 190, New Views of the Magellanic Clouds, Eds. Chu, Y.-H., Suntzeff, N., Hesser, J. \& Bohlender, D., 422

Girardi, L., Bertelli, G., Bressan, A., Chiosi, C., Groenewegen, M. A. T., Marigo, P., Salasnich, B. \& Weiss, A., 2002, A\&A, 391, 195

Harris, J. \& Zaritsky, D., 2001, ApJS, 136, 25

Kim, S., Dopita, M. A., Staveley-Smith, L. \& Bessell, M. S., 1999, AJ, 118, 2797

Meaburn, J., 1980, MNRAS, 192, 365

Mizuno, N. et al., 2001, PASJ, 53, 971

Olsen, K. A. G., Hodge, P. W., Wilcots, E. M. \& Pastwick, L., 1997, ApJ, 475, 545

Westerlund, B. E. \& Mathewson, D. S., 1966, MNRAS, 131, 371

Zaritsky, D., Harris, J., Thompson, I. B., Grebel, E. K. \& Massey, P., 2002, AJ, 123, 855

Zaritsky, D., Harris, J., Thompson, I. B. \& Grebel, E. K., 2004, AJ, 128,1606 